\title{
Homotopy Analysis of the Radiation Effect on MHD Flow with Heat and Mass Transfer due to a Point Sink
}

\author{
C. N. Guled and B. B. Singh \\ Department of Mathematics, Dr. Babasaheb Ambedkar Technological University, Lonere, Raigad District 402103, India
}

Correspondence should be addressed to C. N. Guled; chandrakant.guled@gmail.com

Received 4 June 2016; Revised 8 September 2016; Accepted 27 September 2016

Academic Editor: Alkesh Punjabi

Copyright (C) 2016 C. N. Guled and B.B. Singh. This is an open access article distributed under the Creative Commons Attribution License, which permits unrestricted use, distribution, and reproduction in any medium, provided the original work is properly cited.

\begin{abstract}
An analytical solution of the magnetohydrodynamic, steady, and incompressible laminar boundary layer flow in the presence of heat and mass transfer as well as magnetic field on a cone due to a point sink by using the homotopy analysis method (HAM) has been studied under the radiative fluid properties. The HAM produces an analytical solution of the governing self-similar nonlinear twopoint boundary layer equations. The effects of the suction/injection, magnetic, and radiation parameters over the obtained solution have been discussed. The effects of Prandtl number on temperature and Schmidt number on concentration profiles have also been studied. It has been observed that the temperature profiles exhibit an increasing trend with radiation in case of injection while an opposite trend is observed in case of suction. The results obtained in the present study have also been compared numerically as well as graphically with the corresponding results obtained by using other methods. An excellent agreement has been found between them. The analytical solution obtained by the HAM is very near to the exact solution for a properly selected initial guess, auxiliary, and convergence control parameters and for higher orders of deformations.
\end{abstract}

\section{Introduction}

In many areas of applied engineering and industry, we often come across the boundary layer flow of a steady incompressible laminar fluid flow in the presence of mass transfer and applied magnetic field. This kind of study is of interest for the fields of different branches of innovation, for example, in vortex chambers, magnetohydrodynamic (MHD) power generators, atomic reactors, and geophysical liquid flow. The examination of the boundary layer flow of an electrically conducting fluid on a cone because of a point sink with an applied magnetic field is significant in the investigation of conical nozzle or diffuser-flow problems and it was first of all concentrated on by Choi and Wilhelm [1]. Prior to this problem, Rosenhead [2] studied the same problem in the absence of magnetic field, mass flux diffusion, and heat transfer. Ackerberg [3] presented the series solution for the converging motion of the viscous fluid inside a cone. Takhar et al. [4] extended the same problem for electrically conducting fluid and discussed the heat and mass transfer effects. Eswara et al. [5] investigated the problem for the transient case. Eswara and Bommaiah [6] revisited the problem by taking into account temperature dependent viscosity. Turkyilmazoglu [7] considered the Falkner-Skan flows past stretching boundaries when the momentum and thermal slip boundary conditions are allowed at the boundary. Turkyilmazoglu extended the flow model set-up in a moving convergent channel by Magyari [8] by taking into consideration the momentum slip condition at the wall and found exact analytical solutions for the converging channel, for example, wedge nozzle.

In the context of space technology and processes involving temperatures, the effects of radiation are of vital significance. Recent developments in hypersonic flights, missile reentry, rocket combustion chambers, power plants for interplanetary flight, and gas cooled thermal reactors have focused their attention on thermal radiation as a mode of energy transfer. As a consequence of this, Vyas and Rai [9] made an elaborate analysis of the radiative flow inside a circular cone due to a point sink at the vertex of the cone. It is here worth mentioning that, unlike convection/conduction, the radiative heat transfer mechanism is rather more complex. However, some reasonable approximations have been found 
satisfactory to make the radiative systems solvable. The works of Sparrow and Cess [10] and Howell [11] describe the essentials of the radiative heat transfer. Many other pertinent radiative heat transfer studies for different configurations have also been reported by authors like Plumb et al. [12], Hossain and Takhar [13], Raptis [14], Sedeek and Salem [15], Al-Odat et al. [16], Prasad et al. [17], Mukhopadhyay [18], Vyas and Srivastava [19], Vyas and Ranjan [20], Chauhan and Kumar [21], Baoku et al. [22], Babu et al. [23], and so forth.

The objective of the present paper is to extend the work of Takhar et al. [4] by taking into account the radiative properties of the fluid at the wall. The flow problem which is governed by nonlinear equations with two-point boundary conditions has been solved by using HAM. Using the recursive method derived by Liao [24, 25], rigorous recursive formulae have been developed. Symbolic computation software and high performance computers have been used to derive the analytic solutions. The flow characteristics have been analyzed, and the results have been compared with those of [4] by setting the radiation parameter as zero.

In 1992, Liao [26] investigated the homotopy analysis method (HAM). The strength of HAM is that it leads to convergent analytic series solutions of strongly nonlinear problems faster than any other existing methods, independent of small or large physical parameter/s involved in the problem [27]. This behaviour of HAM makes it a superior technique to the conventional perturbation methods. The methods such as Adomian decomposition method [28-30], $\delta$-expansion method [31], and Lyapunov artificial small parameter method [32] may not be valid for strong nonlinear problems due to the divergent nature of their obtained solution series. Liao [33], indeed, showed that HAM is the general case and the Adomian decomposition method, $\delta$-expansion method and Lyapunov artificial small parameter method are the special cases of HAM. Moreover, He's homotopy perturbation method (HPM) $[34,35]$ is also a special case of the HAM (cf. Liao [36]). Actually, for some auxiliary linear operators, the traditional HPM turns out to be the Taylor series expansion (cf. Turkyilmazoglu [37]).

The HAM has been applied to an extensive variety of nonlinear problems in science and engineering ever since it was first introduced in 1992. The problems of viscous flows of non-Newtonian fluids that have been mainly tackled by Hayat and his coworkers [38-40] and problems of heat transfer $[41,42]$ are some of the examples of applications of HAM. Even a much wider range of applications of HAM can be found in [27].

\section{Governing Equations}

We consider here the boundary layer flow of a fluid which is electrically conducting. The flow is assumed to be steady, laminar, incompressible, and axisymmetric in a circular cone having three-dimensional sink at the vertex (Figure 1).

Here $B_{0}$ is the magnetic field applied in $z$-direction and in comparison with the fluid; it is fixed. With a specific end goal to disregard the induced magnetic field as compared to the applied magnetic field, the magnetic Reynolds number is considered to be small. The temperature and concentration

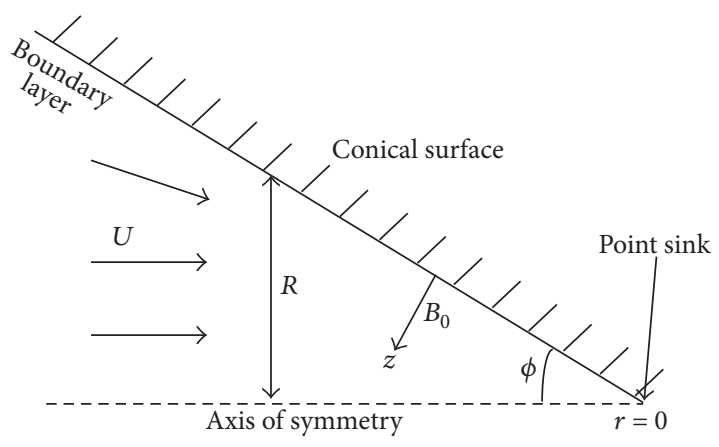

FIGURE 1: Schematic diagram of the flow model.

of the wall and the free stream are preserved at constant level. Importance has been given for studying the effects of suction/injection, magnetic, and radiation parameters and hence they have been included in the analysis. On the other hand, the dissipation terms and the Hall effect have been neglected. It is also considered that the injected gas and the boundary layer gas both possess the same physical properties and are perfect gases. Also, the static temperature of injected gas is equal to the wall temperature. The basic boundary layer equations for the MHD flow due to point sink, including the radiative effect of the fluid at the wall, are (cf. Takhar et al. [4] and Vyas and Rai [9])

$$
\begin{aligned}
(r u)_{r}+(r w)_{z} & =0, \\
u u_{r}+w u_{z} & =-\rho^{-1} p_{r}+v u_{z z}-\rho^{-1} \sigma B_{0}^{2} u, \\
u T_{r}+w T_{z} & =\alpha T_{z z}-\frac{1}{\rho C_{p}} \frac{\partial q_{r}}{\partial z}, \\
u C_{r}+w C_{z} & =D C_{z z},
\end{aligned}
$$

where

$$
-\rho^{-1} p_{r}=U U_{r}+\rho^{-1} \sigma B_{0}^{2} U, \quad U=-\frac{m_{1}}{r^{2}}, m_{1}>0 .
$$

Here the subscripts $r$ and $z$ denote derivatives with respect to $r$ and $z$, respectively, and $p$ represents the static pressure.

The boundary conditions are given by

$$
\begin{gathered}
u(r, 0)=0, \\
w(r, 0)=w_{w}, \\
T(r, 0)=T_{w}, \\
C(r, 0)=C_{w}, \\
u(r, \infty)=U, \\
T(r, \infty)=T_{\infty}, \\
C(r, \infty)=C_{\infty} .
\end{gathered}
$$

We now use the following similarity transformations in (1)(6):

$$
\eta=\frac{m_{1}{ }^{1 / 2} z}{\left(2 v r^{2}\right)^{1 / 2}}
$$




$$
\begin{aligned}
& r u=\Psi_{z}, \\
& r w=-\Psi_{r}, \\
& \Psi=-\left(2 m_{1} \nu r\right)^{1 / 2} f \\
& u=U f^{\prime}(\eta) \\
& w=\left(\frac{m_{1} \nu}{2 r^{3}}\right)^{1 / 2}\left(f-3 \eta f^{\prime}\right) \\
& \frac{T-T_{\infty}}{T_{w}-T_{\infty}}=g(\eta), \\
& \frac{C-C_{\infty}}{C_{w}-C_{\infty}}=G(\eta) \text {, } \\
& M=\frac{2 \sigma B_{0}^{2} r^{3}}{m_{1} \rho}, \\
& \operatorname{Pr}=\frac{\nu}{\alpha} \\
& \mathrm{Sc}=\frac{v}{D}, \\
& K_{w}=w_{w}\left(\frac{2 r^{3}}{m_{1} v}\right)^{1 / 2} \\
& \alpha=\frac{\kappa}{\rho C_{p}}, \\
& N=\frac{16 \gamma^{*} T_{\infty}{ }^{3}}{3 \alpha^{*} \kappa} .
\end{aligned}
$$

Here $r$ is the distance along the cone from the vertex and $u$ is the corresponding velocity component along $r$ direction, whereas $z$ is the distance perpendicular to the cone; $w$ is velocity component along $z$ direction; $R$ is radius of the cone given by $R=r \sin \phi$, where $\phi$ is the semivertical angle of the cone (see Figure 1); $\Psi$ is the dimensional stream function and $f$ represents the corresponding dimensionless stream function; $C$ is the dimensional concentration and $G$ is the corresponding dimensionless concentration; $T$ is the dimensional temperature while $g$ represents dimensionless temperature; $\sigma, \rho$, and $\nu$ are the electrical conductivity, density, and kinematic viscosity, respectively; $\eta$ represents similarity variable; $B_{0}$ stands for magnetic field; $D$ and $\alpha$ represent the binary diffusion coefficient and thermal diffusivity, respectively; $m_{1}$ is the strength of point sink; $M$ is the magnetic parameter; $U$ represents the inviscid flow velocity; $K_{w}$ denotes the mass transfer parameter; $C_{p}$ is the specific heat at constant pressure; $q_{r}$ is the radiative heat flux; $\kappa$ is coefficient of thermal conductivity; $N$ is radiation parameter; $\gamma^{*}$ is the StefanBoltzmann constant, and $\alpha^{*}$ is the mean absorption coefficient.

By using the Rosseland approximation (Brewster [43]), the radiative heat flux is given by

$$
q_{r}=-\frac{4 \gamma^{*}}{3 \alpha^{*}}\left(\frac{\partial T^{4}}{\partial z}\right) \text {. }
$$

Here it should be noted that by using the Rosseland approximation, the present analysis is limited to optically thick fluids. If the temperature differences within the flow are sufficiently small, then (8) can be linearized by expanding $T^{4}$ into the Taylor series about $T_{\infty}$, which after neglecting the higher order terms takes the form

$$
T^{4}=4 T_{\infty}^{3} T-3 T_{\infty}{ }^{4}
$$

In view of the result given in (9) and the similarity transformations given in (7), the continuity equation (1) is satisfied identically, and (2)-(4) get reduced to self-similar equations given by

$$
\begin{array}{r}
f^{\prime \prime \prime}-f f^{\prime \prime}+4\left(1-f^{2}\right)+M\left(1-f^{\prime}\right)=0, \\
(1+N) g^{\prime \prime}-\operatorname{Pr} f g^{\prime}=0, \\
G^{\prime \prime}-\operatorname{Sc} f G^{\prime}=0 .
\end{array}
$$

Also, the boundary conditions (6) get reduced to

$$
\begin{gathered}
f(0)=K_{w}, \\
f^{\prime}(0)=0, \\
f^{\prime}(\infty)=1, \\
g(0)=1, \\
g(\infty)=0, \\
G(0)=1, \\
G(\infty)=0,
\end{gathered}
$$

where Sc and Pr are Schmidt and Prandtl numbers, respectively; the subscripts $w$ denote conditions at the wall and $\infty$ denote conditions in the free stream; and prime denotes derivative with respect to $\eta$.

As pointed out by Rosenhead [2], it is to be noted that the abovementioned boundary layer approximation is not valid in the immediate neighbourhood of the hole (cf. Rosenhead [2], pp. 428). Also for our mathematical analysis, it is remarked that the mass transfer parameter $K_{w}$ is treated as constant (Ref. Takhar et al. [4]). Also, the magnetic parameter $M$ can be treated locally as a constant for a fixed $r$ as it was first considered by Takhar et al. [44] and then by Takhar and Nath [45]. Also in a sink flow, $K_{w}<0$ is referred to as suction and $K_{w}>0$ as injection (cf. Takhar et al. [4] and Schlichting and Gersten [46], pp. 294-298).

\section{Homotopy Analysis}

3.1. Solution for Skin Friction ((10) and (13)). In order to find the analytic solution of (10) along with the boundary conditions given by (13), we first select the linear operator $\mathscr{L}$ as

$$
\mathscr{L}=\frac{\partial^{3}}{\partial \eta^{3}}+\gamma \frac{\partial^{2}}{\partial \eta^{2}},
$$


and we choose $q$ as an embedding parameter. We, now, construct the following zeroth-order deformation equation:

$$
\begin{aligned}
& (1-q) \mathscr{L}\left[f(\eta, \hbar, \gamma, q)-f_{0}(\eta)\right] \\
& =q \hbar \mathscr{N}[f(\eta, \hbar, \gamma, q)] \\
& \quad \eta \in[0,+\infty), \hbar \neq 0, \gamma>0, q \in[0,1]
\end{aligned}
$$

with boundary conditions

$$
\begin{aligned}
f(0, \hbar, \gamma, q) & =K_{w}, \\
f^{\prime}(0, \hbar, \gamma, q) & =0, \\
f^{\prime}(+\infty, \hbar, \gamma, q) & =1,
\end{aligned}
$$

$$
\hbar \neq 0, \gamma>0, q \in[0,1]
$$

where the prime denotes the partial derivative with respect to $\eta$ and

$$
\begin{aligned}
\mathcal{N}[f(\eta, \hbar, \gamma, q)]= & \frac{\partial^{3} f(\eta, \hbar, \gamma, q)}{\partial \eta^{3}} \\
& -f(\eta, \hbar, \gamma, q) \frac{\partial^{2} f(\eta, \hbar, \gamma, q)}{\partial \eta^{2}} \\
& +4\left(1-\left(\frac{\partial f(\eta, \hbar, \gamma, q)}{\partial \eta}\right)^{2}\right) \\
& +M\left(1-\frac{\partial f(\eta, \hbar, \gamma, q)}{\partial \eta}\right) .
\end{aligned}
$$

When $q=0$, we have

$$
\begin{aligned}
\mathscr{L}\left[f(\eta, \hbar, \gamma, 0)-f_{0}(\eta)\right] & =0 \Longrightarrow \\
f(\eta, \hbar, \gamma, 0) & =f_{0}(\eta), \\
\eta & \in[0,+\infty), \hbar \neq 0, \gamma>0
\end{aligned}
$$

and when $q=1$, we have

$$
\begin{aligned}
& 0=\mathcal{N}[f(\eta, \hbar, \gamma, 1)] \Longrightarrow \\
& f(\eta, \hbar, \gamma, 1)=f(\eta), \\
& \eta \in[0,+\infty), \hbar \neq 0, \quad \gamma>0 .
\end{aligned}
$$

Hence, as $q$ varies from 0 to $1, f(\eta, \hbar, \gamma, q)$ varies from initial solution $f_{0}(\eta)$ to the exact solution $f(\eta)$.

Here we choose $f_{0}(\eta)$, the initial guess, such that it satisfies $\mathscr{L}\left(f_{0}(\eta)\right)=0$ and the boundary conditions (13). We select

$$
\begin{aligned}
\mathscr{L}\left[C_{1}+C_{2} \eta+C_{3} e^{-\gamma \eta}\right] & =0, \\
f_{0}(\eta) & =\frac{e^{-\gamma \eta}-1}{\gamma}+K_{w}+\eta,
\end{aligned}
$$

We here assume that the $k$ th-order deformation derivative given by

$$
f_{0}^{[k]}(\eta, \hbar, \gamma)=\left.\frac{\partial^{k} f(\eta, \hbar, \gamma, q)}{\partial q^{k}}\right|_{q=0}, \quad(k \geq 1)
$$

exists. By using (20) and Taylor's formula, we have

$$
f(\eta, \hbar, \gamma, q)=f_{0}(\eta)+\sum_{k=1}^{+\infty}\left[\frac{f_{0}^{[k]}(\eta, \hbar, \gamma)}{k !}\right] q^{k} .
$$

We here assume that both $\hbar$ and $\gamma$ are properly chosen in such a way that the series (25) is convergent at $q=1$. From (21) and (23) at $q=1$, we find the following relationship between known initial solution $f_{0}(\eta)$ and the unknown solution $f(\eta)$ :

$$
f(\eta)=f_{0}(\eta)+\sum_{k=1}^{+\infty} \frac{f_{0}^{[k]}(\eta, \hbar, \gamma)}{k !}=\sum_{k=0}^{+\infty} \varphi_{k}(\eta, \hbar, \gamma)
$$

where we define

$$
\begin{aligned}
& \varphi_{0}(\eta, \hbar, \gamma)=f_{0}(\eta), \\
& \varphi_{k}(\eta, \hbar, \gamma)=\frac{f_{0}^{[k]}(\eta, \hbar, \gamma)}{k !}, \quad k \geq 1 .
\end{aligned}
$$

In order to find the $m$ th-order deformation equation, we first differentiate (17) and (18) $m$ times with respect to $q$ and then we set $q=0$, and finally we divide it by $m$ !, to obtain

$$
\mathscr{L}\left[\varphi_{m}-\chi_{m} \varphi_{m-1}\right]=\mathcal{N}_{m}(\eta)
$$

$$
m \geq 1, \eta \in[0,+\infty)
$$

with the corresponding boundary conditions

$$
\begin{aligned}
& \varphi_{m}(0, \hbar, \gamma)=\varphi_{m}^{\prime}(0, \hbar, \gamma)=\varphi_{m}^{\prime}(+\infty, \hbar, \gamma)=0, \\
& m \geq 1, \hbar \neq 0, \gamma>0, \\
& \mathcal{N}_{1}(\eta)=\hbar\left[\varphi_{0}^{\prime \prime \prime}(\eta, \hbar, \gamma)-\varphi_{0}(\eta, \hbar, \gamma) \varphi_{0}^{\prime \prime}(\eta, \hbar, \gamma)\right. \\
& \left.+4\left(1-\varphi_{0}^{\prime 2}(\eta, \hbar, \gamma)\right)+M\left(1-\varphi_{0}^{\prime}(\eta, \hbar, \gamma)\right)\right], \\
& \mathcal{N}_{m}(\eta)=\hbar\left[\varphi_{m-1}^{\prime \prime \prime}(\eta, \hbar, \gamma)\right. \\
& \quad-\sum_{k=0}^{m-1} \varphi_{m-1-k}(\eta, \hbar, \gamma) \varphi_{k}^{\prime \prime}(\eta, \hbar, \gamma) \\
& +4 \sum_{k=0}^{m-1} \varphi_{k}^{\prime}(\eta, \hbar, \gamma) \varphi_{m-1-k}^{\prime}(\eta, \hbar, \gamma) \\
& \left.+M \varphi_{m-1}^{\prime}(\eta, \hbar, \gamma)\right], \quad m>1,
\end{aligned}
$$

where prime denotes the partial derivative with respect to $\eta$. 
Using (23) and (30), we can first calculate $\mathcal{N}_{1}(\eta)$ and then by solving linear equation (28) with boundary conditions (29), we can find $\varphi_{1}(\eta, \hbar, \gamma)$. In a similar manner, we can calculate $\mathcal{N}_{2}(\eta)$ by using (31) and then find $\varphi_{2}(\eta, \hbar, \gamma)$ and so on. In this way, we solve first few deformation equations by using symbolic software MATHEMATICA and observe that $\varphi_{m}(\eta, \hbar, \gamma)$ can be explicitly expressed as

$$
\varphi_{m}(\eta, \hbar, \gamma)=\sum_{k=0}^{m+1} \psi_{m, k}(\eta, \hbar, \gamma) e^{-k \gamma \eta},
$$

where

$$
\begin{aligned}
& \psi_{0,0}(\eta, \hbar, \gamma)=b_{0,0}^{0}+b_{0,0}^{1} \eta, \\
& \psi_{0,1}(\eta, \hbar, \gamma)=b_{0,1}^{0}, \\
& \psi_{m, 0}(\eta, \hbar, \gamma)=b_{m, 0}^{0}, \quad m \geq 1, \\
& \psi_{m, k}(\eta, \hbar, \gamma)=\sum_{k=0}^{2(m+1)-k} b_{m, k}^{i} \eta^{i},
\end{aligned}
$$

$$
m \geq 1, \quad 1 \leq k \leq m+1 .
$$

The first order of approximation of (10) and (13), calculated by using MATHEMATICA, is

$$
\begin{aligned}
& \varphi_{1}(\eta, \hbar, \gamma)=\frac{5 h e^{-2 \gamma \eta}}{4 \gamma^{3}}-\frac{23 h}{4 \gamma^{3}}+\frac{h}{\gamma}+\frac{h K}{\gamma^{2}}-\frac{h M}{\gamma^{3}} \\
& +e^{-\gamma \eta}\left(\frac{9 h}{2 \gamma^{3}}+\frac{7 \eta h}{\gamma^{2}}-\frac{\eta^{2} h}{2 \gamma}-\frac{h}{\gamma}-\eta h-\frac{h K}{\gamma^{2}}\right. \\
& \left.-\frac{\eta h K}{\gamma}+\frac{h M}{\gamma^{3}}+\frac{\eta h M}{\gamma^{2}}\right) .
\end{aligned}
$$

After knowing the structure (32) of $\varphi_{m}(\eta, \hbar, \gamma)$, we deduce a recurrence formula for the coefficients $b_{m, n}^{k}$ of $\varphi_{m}(\eta, \hbar, \gamma)$ rigorously. Kindly refer to the Appendix for details. Hence, we obtain an analytic solution explicitly for the MHD flow equations with heat and mass transfer due to a point sink, governed by (10) and (13), as

$$
f(\eta)=\lim _{N \rightarrow+\infty} \sum_{k=0}^{N} \varphi_{m}(\eta, \hbar, \gamma) .
$$

The solution (35) in the form of infinite series contains two parameters $\hbar(\hbar \neq 0)$ and $\gamma(\gamma>0)$.

3.2. Convergence of the Analytic Solution. As suggested by Liao [33], the auxiliary parameter $\hbar$ plays a significant role in controlling the convergence and the rate of approximation for the HAM. It is also to be noted that the HAM provides a great deal of flexibility and freedom for choosing appropriate values of $\hbar$ and $\gamma$ so as to ensure the convergence of the solution, obtained in the form of infinite series, to $f(\eta)$. To choose $\hbar$, Liao introduced the concept of $\hbar$-curve that gives an admissible range, called convergence region, for the selection of the

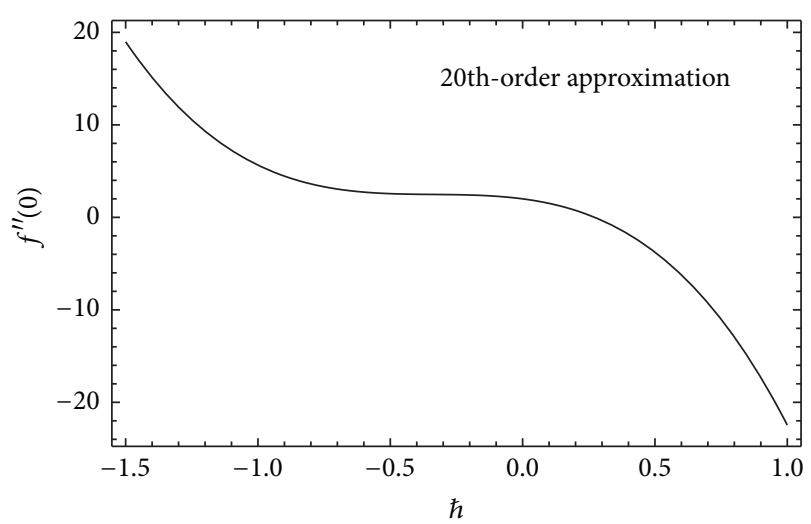

FIgURE 2 : $\hbar$-curve for $M=1, K_{w}=0$, and $\gamma=2$.

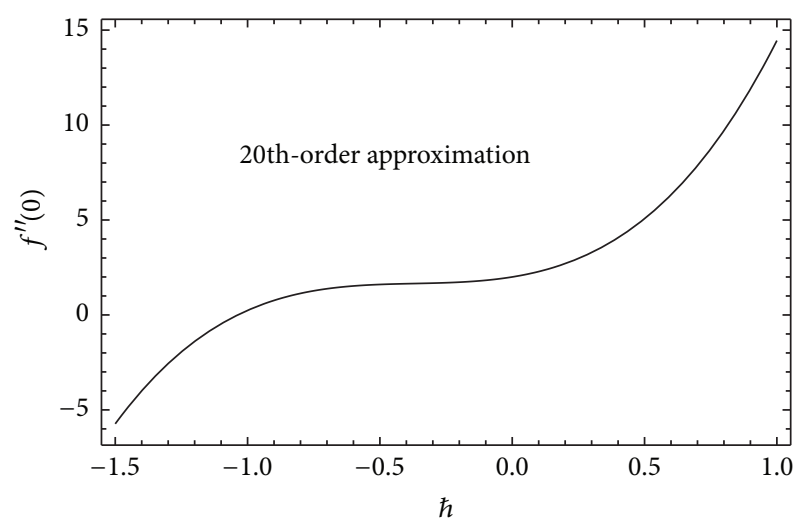

Figure 3 : $\hbar$-curve for $M=1, K_{w}=2$, and $\gamma=2$.

suitable values of $\hbar$. It is here to be emphasized that there exists a value of parameter $\gamma$ for every $\hbar$ belonging to the convergence region which is the most suitable value in the sense that it guarantees the fastest convergence of the given series.

In the present analysis, the values of $f^{\prime \prime}(0)$ for each particular solution of the distinct magnetic parameter $M$ and mass transfer parameter $K_{w}$ have been found by way of selecting suitable values of $\hbar$ and $\gamma$ with the help of $\hbar$-curves given in Figures 2-4. These values have been found after appropriate orders of approximations. These values agree well with the corresponding numerical values of Takhar et al. [4] who tackled the problem by shooting method in conjunction with Runge-Kutta fourth order method. Figure 7 is also an indicator of the fact that $f^{\prime}(\eta)$ obtained from the series given in (35) is also in good agreement with Takhar et al's numerical solution.

It is obvious that if (35) converges, its second-order derivative with respect to $\eta$ at $\eta=0$, say,

$$
\sum_{k=0}^{+\infty} \varphi_{k}^{\prime \prime}(0, \hbar, \gamma)
$$

must converge. By using (A.5), we have its $m$ th-order approximation as

$$
\sigma_{m}=\sum_{k=0}^{m} \varphi_{k}^{\prime \prime}(0, \hbar, \gamma)=\sum_{k=0}^{m} \sum_{n=1}^{k+1} c_{k, n}^{0}
$$




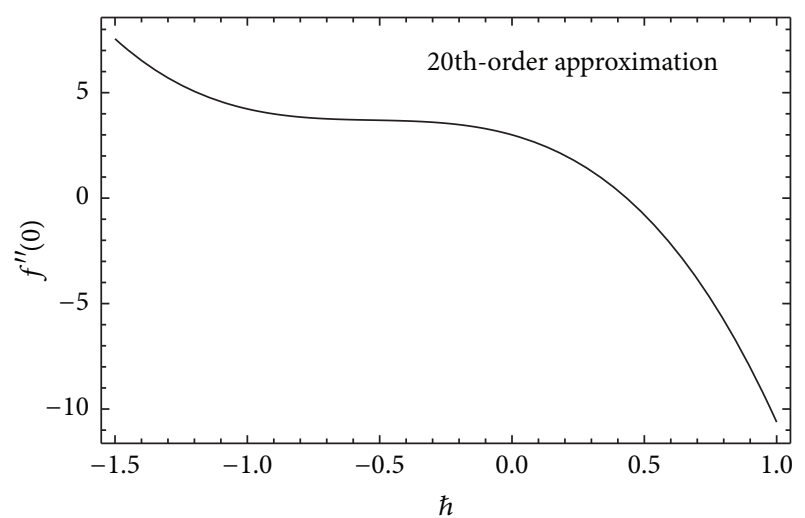

FIGURE 4 : $\hbar$-curve for $M=1, K_{w}=-2$, and $\gamma=3$.

In this context, it is worth mentioning that Turkyilmazoglu [47] has also proposed a new and novel way of finding the optimum value of convergence control parameter $\hbar$ to ensure the convergence of the HAM series in the fastest manner. The proposed method constitutes an alternative to the classical $\hbar$-level curves method (refer to Liao [33]) and the squared residual approach proposed by Liao [48] for the determination of optimal value of the convergence control parameter.

3.3. Solutions for Temperature and Concentration ((11)-(15)). The heat and concentration equations can now easily be solved, for the velocity equation (10) with boundary conditions (13) already possesses a suitable and accurate solution. It is very much precise to obtain the solution of (11) by using boundary conditions (14), as

$$
\begin{gathered}
g(\eta)=1-\frac{\int_{0}^{\eta} e^{\operatorname{Pr}^{*} \int_{0}^{x} f(y) d y} d x}{\int_{0}^{\infty} e^{\operatorname{Pr}^{*} \int_{0}^{x} f(y) d y} d x}, \\
g^{\prime}(\eta)=-\frac{e^{\operatorname{Pr}^{*} \int_{0}^{\eta} f(y) d y}}{\int_{0}^{\infty} e^{\operatorname{Pr}^{*} \int_{0}^{x} f(y) d y} d x} .
\end{gathered}
$$

So, the heat transfer parameter is given by

$$
g^{\prime}(0)=-\frac{1}{\int_{0}^{\infty} e^{\operatorname{Pr}^{*} \int_{0}^{x} f(y) d y} d x},
$$

where $\operatorname{Pr}^{*}=\operatorname{Pr} /(N+1)$. Similarly, the corresponding solution of (12), along with the conditions (15), can be obtained from (38)-(39) simply by replacing $g$ by $G$ and $\operatorname{Pr}^{*}$ by Sc.

\section{Results and Discussion}

By using the recurrence formula (37), the numerical values of the skin-friction parameter $f^{\prime \prime}(0)$ for different values of magnetic parameter $M$ and mass transfer parameter $K_{w}$ (for suction and injection both) have been calculated and are given in Tables 1 and 2. From Table 1, it is obvious that the skinfriction parameter $f^{\prime \prime}(0)$ increases along with the increasing values of the mass suction parameter $K_{w}(<0)$ and magnetic parameter $(M)$. From Table 2 , it is clear that the skin-friction
TABLE 1: Values of $f^{\prime \prime}(0)$ for different values of $M$ and $K_{w}<0$.

\begin{tabular}{cccc}
\hline$M$ & $K_{w}$ & Present & Takhar et al. [4] \\
\hline \multirow{2}{*}{0} & -2 & 3.5211 & 3.5182 \\
& -1 & 2.8517 & 2.8772 \\
0.5 & -2 & 3.6172 & 3.6162 \\
& -1 & 2.9554 & 3.0231 \\
1 & -2 & 3.7098 & 3.7124 \\
& -1 & 3.0542 & 3.1121 \\
\hline
\end{tabular}

TABLE 2: Values of $f^{\prime \prime}(0)$ for different values of $M$ and $K_{w}>0$.

\begin{tabular}{ccccc}
\hline$M$ & $K_{w}$ & Present & Takhar et al. [4] & Rosenhead [2] \\
\hline \multirow{3}{*}{0} & 0 & 2.2721 & 2.2728 & \\
& 1 & 1.7861 & 1.7505 & 2.273 \\
& 2 & 1.4167 & 1.4121 & \\
\hline \multirow{4}{*}{0.5} & 0 & 2.3827 & 2.392 & \\
& 1 & 1.9117 & 1.973 & \\
& 2 & 1.5232 & 1.5529 & \\
1 & 0 & 2.4604 & 2.4552 & \\
& 1 & 2.0158 & 2.0825 & \\
& 2 & 1.6252 & 1.6345 & \\
\hline
\end{tabular}

parameter $f^{\prime \prime}(0)$ increases along with the increasing values of the magnetic parameter $(M)$ but decreases with the increasing values of the mass injection parameter $K_{w}(>0)$. The reason for such a behaviour is that both the suction and the magnetic parameters reduce the thickness of the momentum boundary layer which results in an increase in skin friction. The effect of injection is just opposite.

The numerical values of the skin-friction parameter $f^{\prime \prime}(0)$ for the present case and for the case of Takhar et al. [4] have been given in the Tables 1 and 2 for different values of mass suction/injection and magnetic parameters. These results are in excellent agreement with each other. The skin-friction parameter $f^{\prime \prime}(0)$ and the mass flux diffusion parameter $\left(-g^{\prime}(0)\right)$ have been plotted in Figures 5 and 6 , respectively, for different values of the magnetic and mass transfer parameters. The graphs thus obtained exhibit excellent agreement with the corresponding graphical results obtained by [4] who visited the problem in the absence of radiation parameter $(N)$.

From Figure 6, it is evident that the heat transfer parameter $\left(-g^{\prime}(0)\right)$ decreases for increasing values of the magnetic parameter but increases with increasing values of mass suction $\left(K_{w}<0\right)$. On the other hand, the parameter $-g^{\prime}(0)$ decreases with the increasing values of both the magnetic and mass injection parameters. As a result, the suction $\left(K_{w}<0\right)$ reduces the thermal boundary layer, whereas the injection $\left(K_{w}>0\right)$ and the magnetic parameters increase them.

It is here to be noted that the mass flux diffusion parameter $\left(-G^{\prime}(0)\right)$ is similar to the heat transfer parameter $\left(-g^{\prime}(0)\right)$ in the absence of radiative fluid properties (i.e., for $N=0)$. So, the parameter $-G^{\prime}(0)$ will exhibit similar behaviour as $-g^{\prime}(0)$ shown in Figure 6 . 


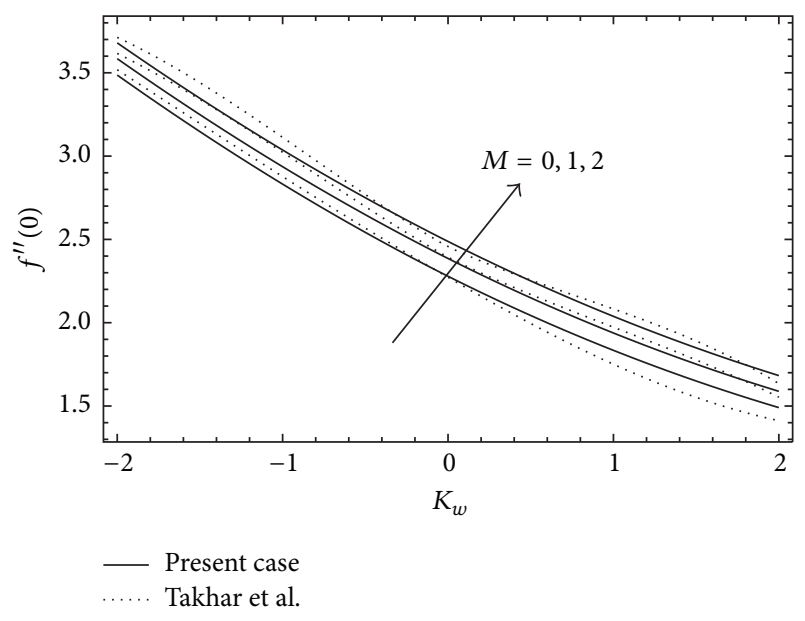

FIGURE 5: Variation of skin friction with $K_{w}$ for $M=0,1,2$ (cf. [4]).

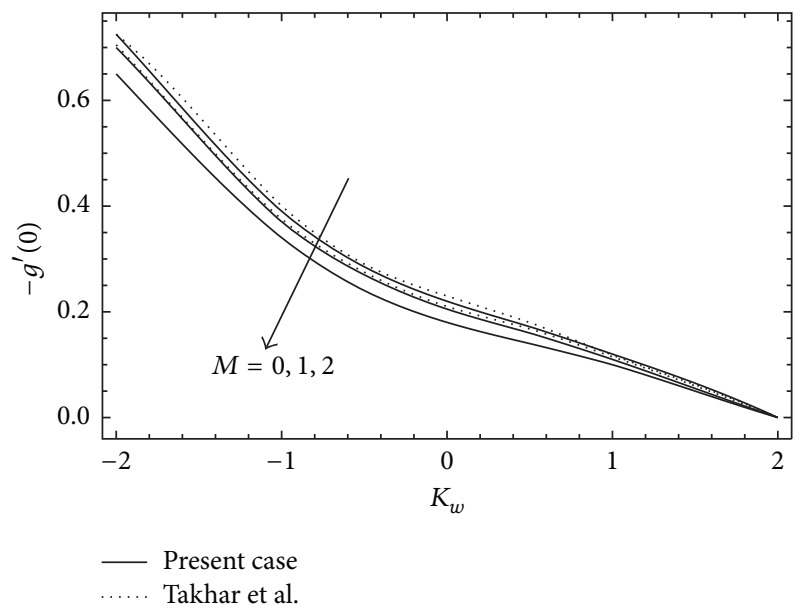

FIGURE 6: Variation of heat transfer and mass diffusion parameters with $K_{w}$ for $M=0,1,2$ and $\mathrm{Pr}=\mathrm{Sc}=0.7, N=0$ (cf. with [4]).

From Figure 7, it is evident that the velocity profiles exhibit an increasing trend with increasing values of the magnetic parameter $(M)$ in both the cases of suction and injection.

From Figure 7, it is also clear that the velocity profiles due to suction $\left(K_{w}<0\right)$ are steeper than those due to injection $\left(K_{w}>0\right)$.

As the velocity increases with an increase in magnetic parameter $(M)$, the thickness of the momentum boundary layer also decreases. This happens due to Lorentz's force arising from the interaction of the magnetic and electric fields during the motion of the electrically conducting fluid.

In Figure 8, the nature of temperature profiles with the Prandtl number $(\mathrm{Pr})$ for a fixed value of magnetic parameter $(M)$ has been studied in the absence of the radiative fluid properties (i.e., $N=0$ ). From Figure 8 , it is clear that the Prandtl number Pr and hence the Schmidt number Sc, respectively, have significant effects on temperature and concentration profiles. Both $\mathrm{Pr}$ and Sc, respectively, increase the temperature and concentration profiles in case of injection. An opposite trend is observed in case of suction.

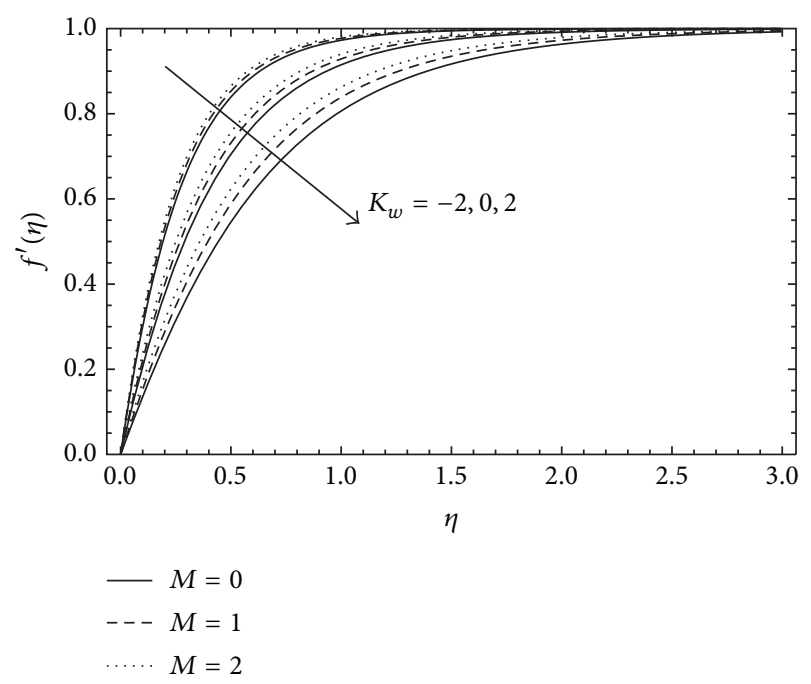

FIGURE 7: Variation of velocity profiles with $K_{w}(-2,0,2)$ for $M=$ $0,1,2$.

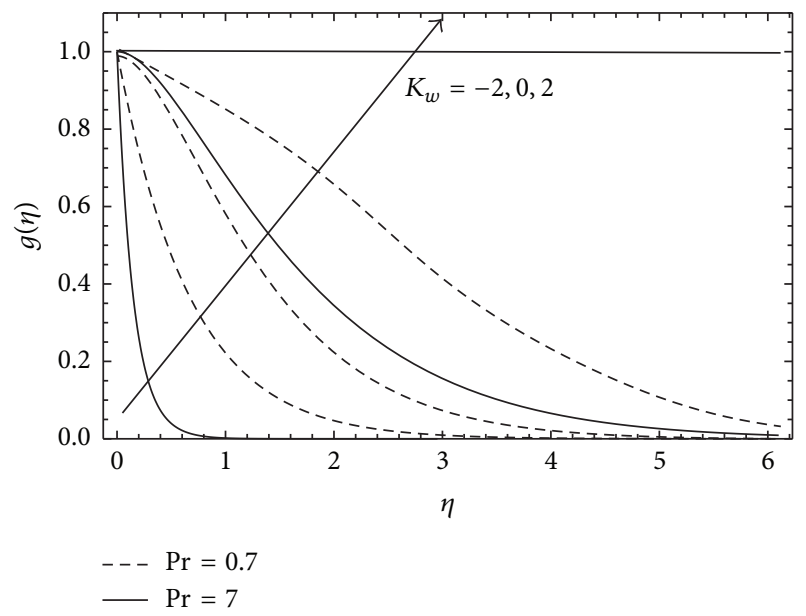

Figure 8: Variation of temperature profiles for $M=1, \operatorname{Pr}(=$ $0.7,7), N=0$, and $K_{w}(=-2,0,2)$.

In Figure 9, the effects of suction and injection parameters on temperature profiles have been studied for fixed values of the magnetic parameter $(M)$, radiation parameter $(N)$, and Prandtl number (Pr). In both the cases of suction and injection, the temperature profiles are showing a decreasing trend along with the similarity variable $\eta$. However, the decreasing trend is more effective in case of suction as compared to that of injection.

Figures 10 and 11 show the variation of temperature profiles with Prandtl number Pr for fixed values of the magnetic and radiation parameters. Figure 10 shows that the temperature profiles exhibit an increasing trend with increasing Pr in case of injection. But an opposite trend is observed in case of suction, as is clear from the Figure 11.

In Figures 12 and 13, the variation of the temperature profiles along with the radiation parameter $(N)$ has been shown for fixed values of magnetic parameter $(M)$ and Prandtl number (Pr) in cases of injection and suction both. 


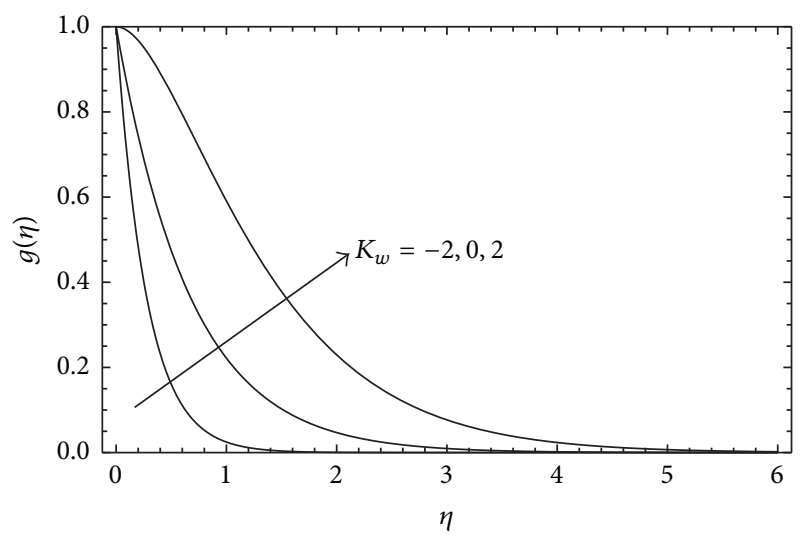

FIGURE 9: Variation of temperature profiles with $K_{w}(=-2,0,2)$ for $M=N=1, \operatorname{Pr}=0.7$.

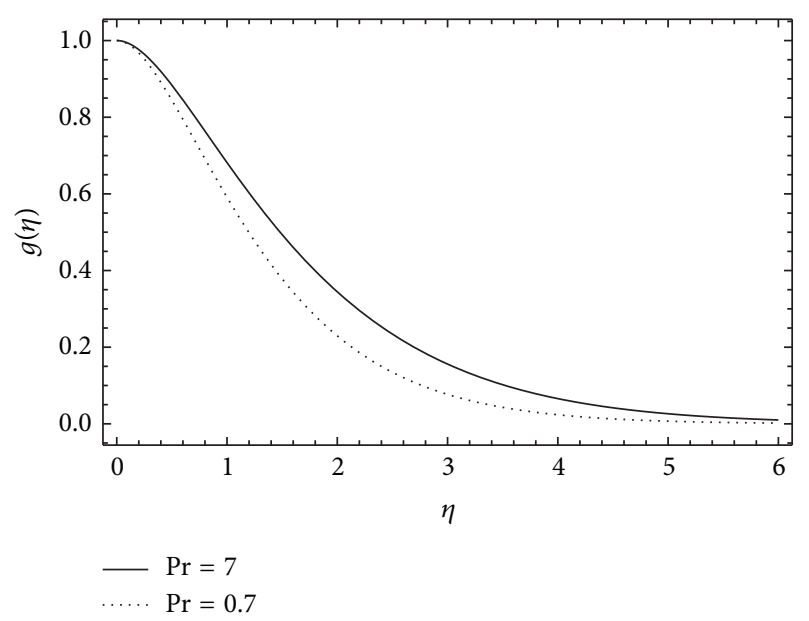

FIGURE 10: Variation of temperature profiles with $\operatorname{Pr}$ for $M=N=1$ and $K_{w}=2$.

From Figure 12, it is obvious that the temperature profiles exhibit an increasing trend with increasing values of $N$ in case of injection. But an opposite trend is observed in case of suction (see Figure 13).

\section{Concluding Remarks}

(1) Our results for $N=0$ have been found in good agreement with those obtained by Takhar et al. [4] which verifies the great potential and validity of the HAM.

(2) The skin-friction increases with increasing magnetic field. The skin friction is greater for suction parameter $\left(K_{w}<0\right)$ as compared to injection parameter $\left(K_{w}>\right.$ $0)$.

(3) The skin-friction $\left(f^{\prime \prime}(0)\right)$, heat transfer parameter $\left(-g^{\prime}(0)\right)$, and mass flux diffusion parameter $\left(-G^{\prime}(0)\right)$ decrease by injection $\left(K_{w}>0\right)$ and increase by suction $\left(K_{w}<0\right)$.

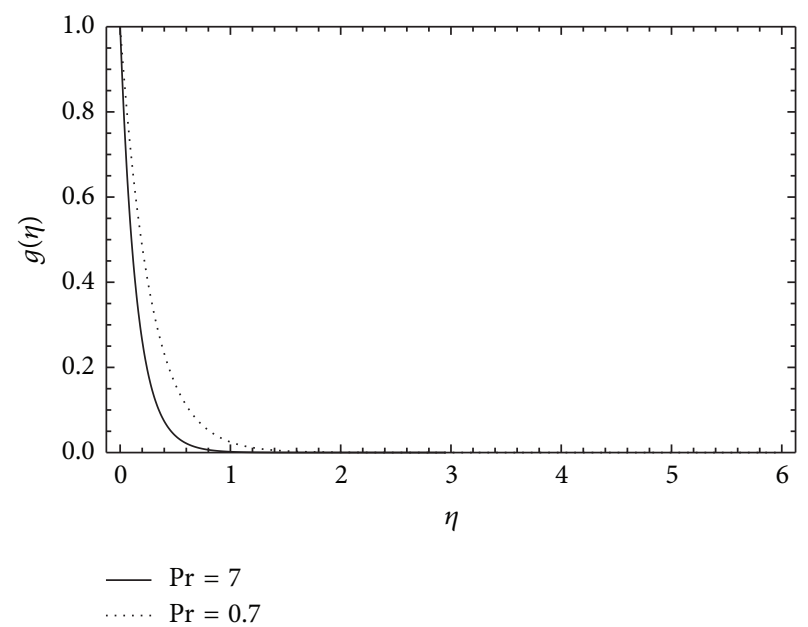

FIgURE 11: Variation of temperature profiles with $\operatorname{Pr}$ for $M=N=1$ and $K_{w}=-2$.

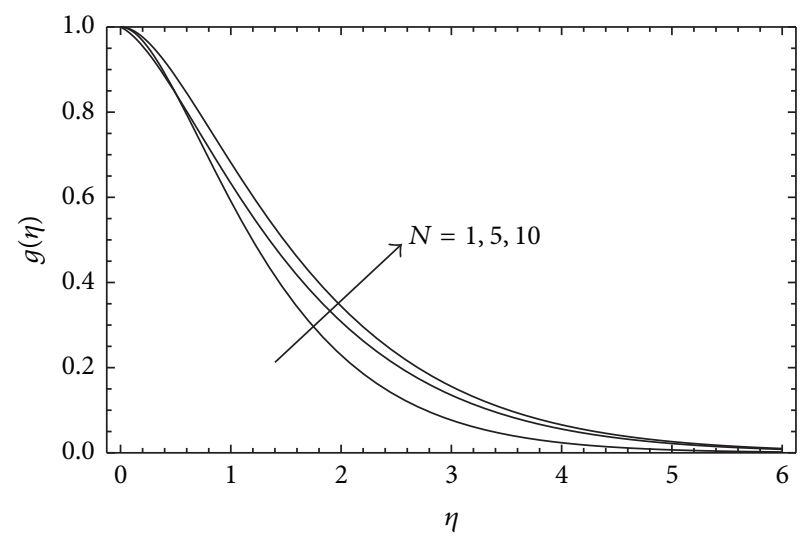

FIGURE 12: Variation of temperature profiles with $N$ for $M=1, \operatorname{Pr}=$ 0.7 , and $K_{w}=2$.

(4) The effect of magnetic field on the skin friction, heat transfer, and mass flux diffusion is less than the effect of mass transfer.

(5) The temperature and concentration profiles get affected by Prandtl number and Schmidt number, respectively.

(6) To get better results, that is, to get better approximations, HAM can offer us large flexibility and great freedom to choose better auxiliary linear operator $(\mathscr{L})$, nonzero auxiliary parameters $(\hbar)$, and spatialscale parameter $(\gamma)$ for satisfying the rule for solution expression and initial approximations.

(7) With the help of high-speed computers and symbolic computation software like MATHEMATICA, Maple, and so forth, the HAM might become more powerful and perfect analytic tool to solve rigorous non-linear problems in science and engineering.

(8) HAM is much superior and accurate method as compared to the shooting method used by Takhar et al. [4] to solve the present problem, for the shooting 


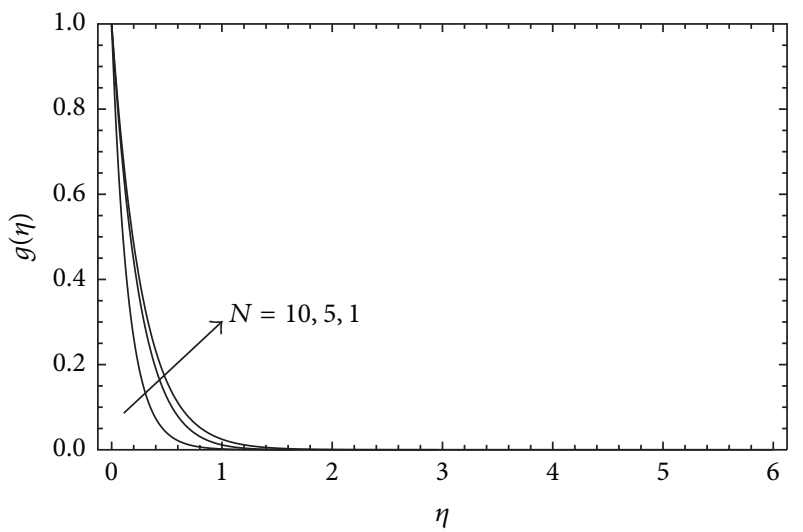

FIGURE 13: Variation of temperature profiles with $N$ for $M=1, \operatorname{Pr}=$ 0.7 , and $K_{w}=-2$.

method gives numerical solution to the problem and HAM gives an analytic solution very near to the exact solution of the problem. So, the results obtained in the present study by using HAM are more credible than those of Takhar et al. [4]. The essence of the shooting method to solve a boundary value problem (BVP) is to convert it into a system of initial value problems where systematic guesses are made for unknown quantities till the end conditions are satisfied. As a consequence of this, this method involves a large number of repetitions if the initial value of the step size ( $h$, say) is far away from the optimal value. So, for the solution of every boundary value problem, there is an optimum step size. For large $h$, the accuracy in the results diminishes, and if $h$ is too small, the roundoff errors would dominate and reduce the accuracy of results.

\section{Appendix}

\section{Derivation of the Coefficients in (32)}

(i) It is to be noted that the real valued function $\psi_{m, k}(\eta, \hbar, \gamma)$ is defined by (33) and the initial guess $f_{0}(\eta)$ defined in (23) has the same structure as (32).

(ii) It can be proved that $\varphi_{m}(\eta, \hbar, \gamma)$ too has the same structure as (32), if we consider that the $(m-1)$ solutions $\varphi_{k}(\eta, \hbar, \gamma)(k=0,1,2,3, \ldots, m-1)$ have the same structure as (32).

We redefine the real function $\psi_{m, k}(\eta, \hbar, \gamma)$ by introducing $\lambda_{m, k}^{i}$ for the sake of ease of calculations as (cf. Liao [25])

$$
\begin{aligned}
\psi_{m, k}(\eta, \hbar, \gamma)=\sum_{k=0}^{2(m+1)-k} \lambda_{m, k}^{i} b_{m, k}^{i} \eta^{i} & \\
& 0 \leq k \leq m+1, \quad m \geq 0,
\end{aligned}
$$

where

$$
\lambda_{m, k}^{i}= \begin{cases}0, & i=j=0, k \geq 2 \\ 0, & i>0, \quad j=0, k \geq 1 \\ 0, & j>i+1 \\ 0, & k>2(i+1)-j \\ 1, & \text { otherwise. }\end{cases}
$$

Now we compute $\varphi_{m}^{\prime}(\eta, \hbar, \gamma), \varphi_{m}^{\prime \prime}(\eta, \hbar, \gamma), \psi_{m, k}^{\prime}(\eta, \hbar, \gamma)$, and $\psi_{m, k}^{\prime \prime}(\eta, \hbar, \gamma)$ as follows:

$$
\begin{aligned}
& \varphi_{m}^{\prime}(\eta, \hbar, \gamma)=\sum_{k=0}^{m+1}\left[\psi_{m, k}^{\prime}-k \gamma \psi_{m, k}\right] e^{-k \gamma \eta}, \\
& \varphi_{m}^{\prime \prime}(\eta, \hbar, \gamma) \\
& =\sum_{k=0}^{m+1}\left[\psi_{m, k}^{\prime \prime}-2 k \gamma \psi_{m, k}^{\prime}+(k \gamma)^{2} \psi_{m, k}\right] e^{-k \gamma \eta}, \\
& \psi_{m, k}^{\prime}(\eta, \hbar, \gamma)=\sum_{i=0}^{2(m+1)-k}(i+1) \lambda_{m, k}^{i+1} b_{m, k}^{i+1} \eta^{i}, \\
& \psi_{m, k}^{\prime \prime}(\eta, \hbar, \gamma)=\sum_{i=0}^{2(m+1)-k}(i+2)(i+1) \lambda_{m, k}^{i+2} b_{m, k}^{i+2} \eta^{i} .
\end{aligned}
$$

By using (A.1)-(A.3), we have

$$
\begin{aligned}
& \varphi_{m}^{\prime}(\eta, \hbar, \gamma)=\sum_{k=0}^{m+1}\left(\sum_{i=0}^{2(m+1)-k} a_{m, k}^{i} \eta^{i}\right) e^{-k \gamma \eta}, \\
& \varphi_{m}^{\prime \prime}(\eta, \hbar, \gamma)=\sum_{k=0}^{m+1}\left(\sum_{i=0}^{2(m+1)-k} c_{m, k}^{i} \eta^{i}\right) e^{-k \gamma \eta}, \\
& \varphi_{m}^{\prime \prime \prime}(\eta, \hbar, \gamma)=\sum_{k=0}^{m+1}\left(\sum_{i=0}^{2(m+1)-k} d_{m, k}^{i} \eta^{i}\right) e^{-k \gamma \eta},
\end{aligned}
$$

where

$$
\begin{aligned}
a_{m, k}^{i}= & (i+1) \lambda_{m, k}^{i+1} b_{m, k}^{i+1}-(k \gamma) \lambda_{m, k}^{i} b_{m, k}^{i}, \\
c_{m, k}^{i}= & (i+1)(i+2) b_{m, k}^{i+2} \lambda_{m, k}^{i+2} \\
& -2(k \gamma)(i+1) b_{m, k}^{i+1} \lambda_{m, k}^{i+1}+(k \gamma)^{2} b_{m, k}^{i} \lambda_{m, k}^{i}, \\
d_{m, k}^{i}= & (i+1) \lambda_{m, k}^{i+1} c_{m, k}^{i+1}-(k \gamma) \lambda_{m, k}^{i} c_{m, k}^{i} .
\end{aligned}
$$

Now we build recurrence formulae for $\varphi_{m-1-k} \varphi_{k}^{\prime \prime}, \varphi_{m-1-k}^{\prime} \varphi_{k}^{\prime}$ in order to find $\mathcal{N}_{m}$. When $0 \leq k \leq m-1$, we get by using (32), (A.2), and (A.5)

$$
\varphi_{m-1-k} \varphi_{k}^{\prime \prime}=\left[\sum_{r=0}^{m-k}\left(\sum_{s=0}^{2(m-k)-r} \lambda_{m-i-k, r}^{s} b_{m-1-k, r}^{s} \eta^{s}\right) e^{-r \gamma \eta}\right]\left[\sum_{j=1}^{k+1}\left(\sum_{i=0}^{2(k+1)-j} c_{k, j}^{i} \eta^{i}\right) e^{-j \gamma \eta}\right]
$$




$$
\begin{aligned}
& =\sum_{j=1}^{k+1} \sum_{r=0}^{m-k}\left(\sum_{i=0}^{2(k+1)-j 2(m-k)-r} \sum_{s=0}^{i} c_{k, j}^{i} \lambda_{m-1-k, r}^{s} b_{m-1-k, r}^{s} \eta^{i+s}\right) e^{-(j+r) \gamma \eta} \\
& =\sum_{n=1}^{m+1} \sum_{j=\max \{1, n-(m-k)\}}^{\min \{n, k+1\}}\left(\sum_{p=0}^{2(m+1)-n} \sum_{i=\max \{0, p-2(m-k)+n-j\}}^{\min \{p, 2(k+1)-j\}} c_{k, j}^{i} \lambda_{m-1-k, n-j}^{p-i} b_{m-1-k, n-j}^{p-i} \eta^{p}\right) e^{-n \gamma \eta} \\
& =\sum_{n=1}^{m+1}\left(\sum_{p=0}^{2(m+1)-n}\left(\sum_{j=\max \{1, n-(m-k)\}}^{\min \{n, k+1\}} \sum_{i=\max \{0, p-2(m-k)+n-j\}}^{\min \{p, 2(k+1)-j\}} c_{k, j}^{i} \lambda_{m-1-k, n-j}^{p-i} b_{m-1-k, n-j}^{p-i}\right) \eta^{p}\right) e^{-n \gamma \eta} .
\end{aligned}
$$

Hence,

$$
\begin{aligned}
-\sum_{k=0}^{m-1} \varphi_{m-1-k} \varphi_{k}^{\prime \prime} & =-\sum_{k=0}^{m-1} \sum_{n=1}^{m+1}\left(\sum_{p=0}^{2(m+1)-n}\left(\sum_{j=\max \{1, n-(m-k)\}}^{\min \{n, k+1\}} \sum_{i=\max \{0, p-2(m-k)+n-j\}}^{\min \{p, 2(k+1)-j\}} c_{k, j}^{i} \lambda_{m-1-k, n-j}^{p^{-i}} b_{m-1-k, n-j}^{p-i}\right) \eta^{p}\right) e^{-n \gamma \eta} \\
& =-\sum_{n=1}^{m+1}\left(\sum_{p=0}^{2(m+1)-n}\left(\sum_{k=0}^{m-1} \sum_{j=\max \{1, n-(m-k)\}}^{\min \{n, k+1\}} c_{i=\max \{0, p-2(m-k)+n-j\}}^{i} \lambda_{k, j}^{p-i} \lambda_{m-1-k, n-j} b_{m-1-k, n-j}^{p-i}\right) \eta^{p}\right) e^{-n \gamma \eta} \\
& =-\sum_{n=1}^{m+1}\left(\sum_{p=0}^{2(m+1)-n} \delta_{m, n}^{p} \eta^{p}\right) e^{-n \gamma \eta},
\end{aligned}
$$

where

$$
\delta_{m, n}^{p}=-\sum_{k=0}^{m-1} \sum_{j=\max \{1, n-(m-k)\}}^{\min \{n, k+1\}} \sum_{i=\max \{0, p-2(m-k)+n-j\}}^{\min \{p, 2(k+1)-j\}} c_{k, j}^{i} \lambda_{m-1-k, n-j}^{p-i} b_{m-1-k, n-j}^{p-i} \quad \forall 1 \leq n \leq m+1
$$

$0 \leq p \leq 2(m+1)-n$.

Here, we point out that $\delta_{n, 1}^{2 m+1}=0$ for all $m \geq 1$. Hence, we have from (A.9) and (A.10) for $m \geq 1$

$$
\begin{aligned}
-\sum_{k=0}^{m-1} \varphi_{m-1-k} \varphi_{k}^{\prime \prime}= & \sum_{n=1}^{m+1}\left(\sum_{p=0}^{2(m+1)-n} \delta_{m, n}^{p} \eta^{p}\right) e^{-n \gamma \eta} \\
= & -e^{-\gamma \eta} \sum_{p=0}^{2 m+1} \delta_{m, 1}^{p} \eta^{p} \\
& -\sum_{n=2}^{m+1}\left(\sum_{p=0}^{2(m+1)-n} \delta_{m, n}^{p} \eta^{p}\right) e^{-n \gamma \eta} \\
= & -e^{-\gamma \eta} \sum_{p=0}^{2 m} \delta_{m, 1}^{p} \eta^{p} \\
& -\sum_{n=2}^{m+1}\left(\sum_{p=0}^{2(m+1)-n} \delta_{m, n}^{p} \eta^{p}\right) e^{-n \gamma \eta}
\end{aligned}
$$

Similarly, we have

$$
4 \sum_{k=0}^{m-1} \varphi_{k}^{\prime} \varphi_{m-1-k}^{\prime}=\sum_{n=0}^{m+1}\left(\sum_{p=0}^{2(m+1)-n} \Delta_{m, n}^{p} \eta^{p}\right) e^{-n \gamma \eta}
$$

where

$$
\begin{aligned}
& \Delta_{m, n}^{p} \\
& =4 \sum_{k=0}^{m-1} \sum_{j=\max \{0, n+k-m\}}^{\min \{n, k+1\}} \sum_{i=\max \{0, p-2(m-k)+n-j\}}^{\min \{p, 2(k+1)-j\}} a_{k, j}^{i} a_{m-1-k, n-j}^{p-i} .
\end{aligned}
$$

Now, (A.12) leads to

$$
\begin{aligned}
4 \sum_{k=0}^{m-1} \varphi_{k}^{\prime} \varphi_{m-1-k}^{\prime}= & \sum_{p=0}^{2 m+1}\left(\Delta_{m, 1}^{p} \eta^{p}\right) e^{-\gamma \eta} \\
& +\sum_{n=2}^{m+1}\left(\sum_{p=0}^{2(m+1)-n} \Delta_{m, n}^{p} \eta^{p}\right) e^{-n \gamma \eta} .
\end{aligned}
$$

But it is observed that $\Delta_{m, 1}^{2 m+1}=0$ for $m \geq 1$. 
So, we have

$$
\begin{aligned}
4 \sum_{k=0}^{m-1} \varphi_{k}^{\prime} \varphi_{m-1-k}^{\prime}= & \sum_{p=0}^{2 m} \Delta_{m, 1}^{p} \eta^{p} e^{-\gamma \eta} \\
& +\sum_{n=2}^{m+1}\left(\sum_{p=0}^{2(m+1)-n} \Delta_{m, n}^{p} \eta^{p}\right) e^{-n \gamma \eta} .
\end{aligned}
$$

Finally, putting all these in (31), $\mathcal{N}_{m}$ becomes

$$
\mathcal{N}_{m}=e^{-\gamma \eta}\left(\sum_{p=0}^{2 m} \Gamma_{m, 1}^{p} \eta^{p}\right)
$$

$$
+\sum_{n=2}^{m+1}\left(\sum_{p=0}^{2(m+1)-n} \Gamma_{m, n}^{p} \eta^{p}\right) e^{-n \gamma \eta}
$$

(A.15) $\quad$ where, for $m \geq 1$,

$$
\begin{aligned}
\Gamma_{m, 1}^{p} & =\hbar\left(d_{m-1,1}^{p}+\delta_{m, 1}^{p}+\Delta_{m, 1}^{p}+M a_{m-1,1}^{p}\right) \\
& \forall 0 \leq p \leq 2 m-1 \\
\Gamma_{m, 1}^{2 m}=\hbar\left(\delta_{m, 1}^{2 m}+\Delta_{m, 1}^{2 m}\right) & \\
\Gamma_{m, m+1}^{p}=\hbar\left(\delta_{m, m+1}^{p}+\Delta_{m, m+1}^{p}\right) & \forall 0 \leq p \leq m+1
\end{aligned}
$$

and, for $2 \leq n \leq m$,

$$
\Gamma_{m, n}^{p}= \begin{cases}\hbar\left(d_{m-1, n}^{p}+\delta_{m, n}^{p}+\Delta_{m, n}^{p}+M a_{m-1, n}^{p}\right), & 0 \leq p \leq 2 m-n \\ \hbar\left(\delta_{m, n}^{p}+\Delta_{m, n}^{p}\right), & 2 m-n+1 \leq p \leq 2 m-n+2 \\ 0, & \text { otherwise. }\end{cases}
$$

Hence, by substituting (A.16) into (28), we have the $m$ th-order deformation equation

$$
\begin{aligned}
\mathscr{L}\left[\varphi_{m}-\chi_{m} \varphi_{m-1}\right]= & e^{-\gamma \eta}\left(\sum_{p=0}^{2 m} \Gamma_{m, 1}^{p} \eta^{p}\right) \\
& +\sum_{n=2}^{m+1}\left(\sum_{p=0}^{2(m+1)-n} \Gamma_{m, n}^{p} \eta^{p}\right) e^{-n \gamma \eta} .
\end{aligned}
$$

That is,

$$
\begin{aligned}
\mathscr{L}\left[Y_{m}\right]= & e^{-\gamma \eta}\left(\sum_{p=0}^{2 m} \Gamma_{m, 1}^{p} \eta^{p}\right) \\
& +\sum_{n=2}^{m+1}\left(\sum_{p=0}^{2(m+1)-n} \Gamma_{m, n}^{p} \eta^{p}\right) e^{-n \gamma \eta},
\end{aligned}
$$

where $Y_{m}=\varphi_{m}-\chi_{m} \varphi_{m-1}$ and $\mathscr{L}$ is given by (16).

Hence,

$$
\begin{aligned}
Y_{m} & =C_{1}^{m}+C_{2}^{m} \eta+C_{3}^{m} e^{-\gamma \eta}+\sum_{p=0}^{2 m} \Gamma_{m, 1}^{p} L^{-1}\left(\eta^{p} e^{-\gamma \eta}\right) \\
& +\sum_{n=2}^{m+1} \sum_{p=0}^{2(m+1)-n} \Gamma_{m, n}^{p} L^{-1}\left(\eta^{p} e^{-n \gamma \eta}\right)=C_{1}^{m}+C_{2}^{m} \eta \\
& +C_{3}^{m} e^{-\gamma \eta}+\sum_{p=0}^{2 m} \Gamma_{m, 1}^{p} \frac{1}{D^{2}(D+\gamma)}\left(\eta^{p} e^{-\gamma \eta}\right) \\
& +\sum_{n=2}^{m+1} \sum_{p=0}^{2(m+1)-n} \Gamma_{m, n}^{p} \frac{1}{D^{2}(D+\gamma)}\left(\eta^{p} e^{-n \gamma \eta}\right)=C_{1}^{m} \\
& +C_{2}^{m} \eta+C_{3}^{m} e^{-\gamma \eta}+\sum_{p=0}^{2 m} \Gamma_{m, 1}^{p} \frac{1}{D^{2}} e^{-\gamma \eta}
\end{aligned}
$$

$$
\begin{aligned}
& \cdot \int\left(e^{\gamma \eta} \eta^{p} e^{-\gamma \eta}\right) d \eta+\sum_{n=2}^{m+1} \sum_{p=0}^{2(m+1)-n} \Gamma_{m, n}^{p} \frac{1}{D^{2}} e^{-n \gamma \eta} \\
& \cdot \int\left(e^{n \gamma \eta} \eta^{p} e^{-n \gamma \eta}\right) d \eta=C_{1}^{m}+C_{2}^{m} \eta+C_{3}^{m} e^{-\gamma \eta} \\
& +\sum_{p=0}^{2 m} \Gamma_{m, 1}^{p} \frac{1}{D^{2}} \frac{\eta^{p+1} e^{-\gamma \eta}}{p+1}+\sum_{n=2}^{m+1} \sum_{p=0}^{2(m+1)-n} \Gamma_{m, n}^{p} \frac{1}{D^{2}} e^{-n \gamma \eta}
\end{aligned}
$$$$
\cdot\left(\sum_{j=0}^{p} \frac{p !}{j !} \frac{\eta^{j}}{((n-1) \eta)^{p-j+1}}\right)=C_{1}^{m}+C_{2}^{m} \eta
$$$$
+C_{3}^{m} e^{-\gamma \eta}+\sum_{p=0}^{2 m} \Gamma_{m, 1}^{p} \iint \frac{\eta^{p+1} e^{-\gamma \eta}}{p+1} d \eta d \eta
$$$$
+\sum_{n=2}^{m+1} \sum_{p=0}^{2(m+1)-n} \Gamma_{m, n}^{p}
$$$$
\cdot \iint e^{-n \gamma \eta} \sum_{j=0}^{p} \frac{p !}{j !} \frac{\eta^{j}}{((n-1) \eta)^{p-j+1}} d \eta d \eta=C_{1}^{m}
$$$$
+C_{2}^{m} \eta+C_{3}^{m} e^{-\gamma \eta}
$$$$
+e^{-\gamma \eta}\left[\sum_{p=0}^{2 m} \Gamma_{m, 1}^{p} \mu_{m, 1}^{p}+\sum_{k=1}^{2 m+1} \eta^{k}\left(\sum_{q=k-1}^{2 m} \Gamma_{m, 1}^{p} \mu_{1, k}^{p}\right)\right]
$$$$
-\sum_{n=2}^{m+1} e^{-n \gamma \eta}\left[\sum_{k=0}^{2(m+1)-n} \eta^{k}\left(\sum_{p=k}^{2(m+1)-n} \Gamma_{m, n}^{p} \mu_{n, k}^{p}\right)\right] \text {, }
$$ 
where $C_{1}^{m}, C_{2}^{m}$, and $C_{3}^{m}$ are the integral constants:

$$
\begin{gathered}
\mu_{1, k}^{p}=\frac{p !(p-k+2)}{k ! \gamma^{p-k+3}}, \quad 0 \leq k \leq p+1, p \geq 0, \\
\mu_{n, k}^{p}=\frac{p !(p-k+2)}{k !(n-1)^{p-k+1} \gamma^{p-k+3}}\left(1-\left(1-\frac{1}{n}\right)^{p-k+1}\right. \\
\left.\cdot\left[(p-k+2)-(p-k+1)\left(1-\frac{1}{n}\right)\right]\right), \\
0 \leq k \leq p, p \geq 0, n \geq 2 .
\end{gathered}
$$

Now applying boundary conditions $f(0)=K_{w}, f^{\prime}(0)=0$, and $f^{\prime}(\infty)=1$ we get

$$
\begin{aligned}
C_{1}^{m} & =\sum_{p=0}^{2 m} \Gamma_{m, 1}^{p}\left(\gamma^{-1} \mu_{1,1}^{p}-\mu_{1,0}^{p}\right)+\sum_{n=2}^{m+1}\left[n \Gamma_{m, n}^{0} \mu_{n, 0}^{0}\right. \\
& \left.+\sum_{p=1}^{2(m+1)-n} \Gamma_{m, n}^{p}\left(n \mu_{n, 0}^{p}-\gamma^{-1} \mu_{n, 1}^{p}\right)\right], \\
C_{2}^{m} & =0, \\
C_{3}^{m} & =-C_{1}^{m}-\sum_{p=0}^{2 m} \Gamma_{m, 1}^{p} \mu_{1,0}^{p}+\sum_{n=2}^{m+1} \sum_{p=0}^{2(m+1)-n} \Gamma_{m, n}^{p} \mu_{n, 0}^{p} .
\end{aligned}
$$

This shows that $\varphi_{m}$ has the same structure as (32) and hence the related coefficients $b_{m, n}^{k}$ can be obtained by the following recurrence relations:

$$
\begin{aligned}
b_{m, 0}^{0} & =\chi_{m} b_{m-1,0}^{0}-\gamma^{-1} \sum_{p=0}^{2 m} \Gamma_{m, 1}^{p} \mu_{1,1}^{p} \\
& -\sum_{n=2}^{m+1}\left[(n-1) \Gamma_{m, n}^{0} \mu_{n, 0}^{0}\right. \\
& \left.+\sum_{p=1}^{2(m+1)-n} \Gamma_{m, n}^{p}\left(n \mu_{n, 0}^{p}-\mu_{n, 0}^{p}-\gamma^{-1} \mu_{n, 1}^{p}\right)\right] \\
b_{m, 0}^{1} & =0, \\
b_{m, 1}^{0} & =\chi_{m} b_{m-1,1}^{0}+\gamma^{-1} \sum_{p=0}^{2 m} \Gamma_{m, 1}^{p} \mu_{1,1}^{p}+\sum_{n=2}^{m+1}\left[n \Gamma_{m, n}^{0} \mu_{n, 0}^{0}\right. \\
& \left.\sum_{p=1}^{2(m+1)-n} \Gamma_{m, n}^{p}\left(n \mu_{n, 0}^{p}-\gamma^{-1} \mu_{n, 1}^{p}\right)\right] \\
b_{m, 1}^{k} & =\sum_{p=k-1}^{k} \Gamma_{m, 1}^{p} \mu_{1, k}^{p} 2 m \leq k \leq 2 m+1, \\
& =\chi_{m} b_{m-1,1}^{k}+\sum_{p=k-1}^{2 m} \Gamma_{m, 1}^{p} \mu_{1, k}^{p}, 1 \leq k \leq 2 m-1,
\end{aligned}
$$

$$
\begin{aligned}
& b_{m, n}^{k}=\chi_{m} b_{m-1, n}^{k}-\sum_{p=k}^{2(m+1)-n} \Gamma_{m, n}^{p} \mu_{n, k}^{p}, \\
& \quad 0 \leq k \leq 2 m-n, 2 \leq n \leq m, \\
& b_{m, n}^{k}=-\sum_{p=k}^{2(m+1)-n} \Gamma_{m, n}^{p} \mu_{n, k}^{p}, \\
& \quad 2 m-n+1 \leq k \leq 2 m-n+2,2 \leq n \leq m, \\
& b_{m, m+1}^{k}=-\sum_{p=k}^{m+1} \Gamma_{m, m+1}^{p} \mu_{m+1, k}^{p}, \quad 0 \leq k \leq m+1 .
\end{aligned}
$$

(iii) In (i), we remarked that the initial guess approximation $\varphi_{0}(\eta)=f_{0}(\eta)$ has the same structure as (32). In (ii), we derived all the recurrence formulae (A.1)-(A.24) and also we have proved that $\varphi_{m}(\eta, \hbar, \gamma)(m \geq 1)$ too should have the same structure as (32), if we consider that the first $(m-1)$ solutions $\varphi_{k}(\eta, \hbar, \gamma)(k=0,1,2,3, \ldots, m-1)$ have the same structure as (32). Hence, from (i) and (ii), all $\varphi_{k}(\eta, \hbar, \gamma)(k \geq 0)$ have the same structure as (32). Along these lines, we can figure all coefficients $b_{m, n}^{k}$ in a steady progression by utilizing the initial four known coefficients:

$$
\begin{aligned}
& b_{0,0}^{0}=K_{w}-\gamma^{-1}, \\
& b_{0,0}^{1}=1, \\
& b_{0,1}^{0}=-\gamma^{-1} \\
& b_{0,1}^{1}=0
\end{aligned}
$$

which are dictated by the initial guess estimation $f_{0}(\eta)$ characterized by (13).

\section{Competing Interests}

The authors declare that there is no conflict of interests regarding the publication of this paper.

\section{References}

[1] S. H. Choi and H. E. Wilhelm, "Self-similar magnetohydrodynamic diffuser flows with induced magnetic fields," Physics of Fluids, vol. 20, no. 11, pp. 1821-1826, 1977.

[2] L. Rosenhead, Laminar Boundary Layers, Clarendon Press, Oxford, UK, 1963.

[3] R. C. Ackerberg, "The viscous incompressible flow inside a cone," Journal of Fluid Mechanics, vol. 21, pp. 47-81, 1965.

[4] H. S. Takhar, C. D. Surma Devi, and G. Nath, "MHD flow with heat and mass transfer due to a point sink," Indian Journal of Pure and Applied Mathematics, vol. 17, no. 10, pp. 1242-1247, 1986.

[5] A. T. Eswara, S. Roy, and G. Nath, "Unsteady MHD forced flow due to a point sink," Acta Mechanica, vol. 145, no. 1-4, pp. 159$172,2000$.

[6] A. T. Eswara and B. C. Bommaiah, "The effect of variable viscosity on laminar flow due to a point sink," Indian Journal of Pure and Applied Mathematics, vol. 35, no. 6, pp. 811-815, 2004. 
[7] M. Turkyilmazoglu, "Exact multiple solutions for the slip flow and heat transfer in a converging channel," Journal of Heat Transfer, vol. 137, no. 10, Article ID 101301, 2015.

[8] E. Magyari, "Backward boundary layer heat transfer in a converging channel," Japan Society of Fluid Mechanics, vol. 39, no. 6, pp. 493-504, 2007.

[9] P. Vyas and A. Rai, "Radiative variable fluid properties flow due to a point sink inside a cone filled with porous medium," Applied Mathematical Sciences, vol. 6, no. 87, pp. 4307-4317, 2012.

[10] E. M. Sparrow and R. D. Cess, Radiation Heat Transfer, Brooke/ Cole, Belmont, Calif, USA, 1970.

[11] J. R. Howell, "Radiative transfer in porous media," in Handbook of Porous Media, K. Vafai, Ed., pp. 663-698, CRC Press, New York, NY, USA, 2000.

[12] O. A. Plumb, J. S. Huenefeld, and E. J. Eschbach, “The effect of crossbow and radiation on natural convection from vertical heated surfaces in saturated porous media," in Proceedings of the AIAA 16th Thermophysics Conference, Polo Alto, Calif, USA, 1981.

[13] M. A. Hossain and H. S. Takhar, "Radiation effect on mixed convection along a vertical plate with uniform surface temperature," Heat and Mass Transfer, vol. 31, no. 4, pp. 243-248, 1996.

[14] A. Raptis, "Radiation and free convection flow through a porous medium," International Communications in Heat and Mass Transfer, vol. 25, no. 2, pp. 289-295, 1998.

[15] M. A. Seddeek and A. M. Salem, "Laminar mixed convection adjacent to vertical continuously stretching sheets with variable viscosity and variable thermal diffusivity," Heat and Mass Transfer, vol. 41, no. 12, pp. 1048-1055, 2005.

[16] M. Q. Al-Odat, F. M. S. Al-Hussien, and R. A. Damseh, "Influence of radiation on mixed convection over a wedge in non-Darcy porous medium," Forschung im Ingenieurwesen, vol. 69, no. 4, pp. 209-215, 2005.

[17] V. R. Prasad, N. B. Reddy, and R. Muthucumaraswamy, "Radiation and mass transfer effects on two-dimensional flow past an impulsively started infinite vertical plate," International Journal of Thermal Sciences, vol. 46, no. 12, pp. 1251-1258, 2007.

[18] S. Mukhopadhyay, "Effects of radiation and variable fluid viscosity on flow and heat transfer along a symmetric wedge," Journal of Applied Fluid Mechanics, vol. 2, no. 2, pp. 29-34, 2009.

[19] P. Vyas and N. Srivastava, "Radiative MHD flow over a non-isothermal stretching sheet in a porous medium," Applied Mathematical Sciences, vol. 4, no. 50, pp. 2475-2484, 2010.

[20] P. Vyas and A. Ranjan, "Dissipative MHD boundary-layer flow in a porous medium over a sheet stretching nonlinearly in the presence of radiation," Applied Mathematical Sciences, vol. 4, no. 61-64, pp. 3133-3142, 2010.

[21] D. S. Chauhan and V. Kumar, "Radiation effects on mixed convection flow and viscous heating in a vertical channel partially filled with a porous medium," Tamkang Journal of Science and Engineering, vol. 14, no. 2, pp. 97-106, 2011.

[22] I. G. Baoku, C. Israel-Cookey, and B. I. Olajuwon, "Influence of thermal radiation on a transient MHD couette flow through a porous medium," Journal of Applied Fluid Mechanics, vol. 5, no. 1, pp. 81-87, 2012.

[23] P. R. Babu, J. A. Rao, and S. Sheri, "Radiation effect on Mhd heat and mass transfer flow over a shrinking sheet with mass suction," Journal of Applied Fluid Mechanics, vol. 7, no. 4, pp. 641650, 2014.

[24] S.-J. Liao, "An explicit, totally analytic approximate solution for Blasius' viscous flow problems," International Journal of NonLinear Mechanics, vol. 34, no. 4, pp. 759-778, 1999.
[25] S.-J. Liao, "A uniformly valid analytic solution of two-dimensional viscous flow over a semi-infinite flat plate," Journal of Fluid Mechanics, vol. 385, pp. 101-128, 1999.

[26] S. J. Liao, The proposed homotopy analysis technique for the solution of nonlinear problems [Ph.D. thesis], Shanghai Jiao Tong University, 1992.

[27] S. J. Liao, "Notes on the homotopy analysis method: some definitions and theorems," Communications in Nonlinear Science and Numerical Simulation, vol. 14, no. 4, pp. 983-997, 2009.

[28] G. Adomian, "Nonlinear stochastic differential equations," Journal of Mathematical Analysis and Applications, vol. 55, no. 2, pp. 441-452, 1976.

[29] G. Adomian, "A review of the decomposition method and some recent results for nonlinear equations," Computers \& Mathematics with Applications, vol. 21, no. 5, pp. 101-127, 1991.

[30] R. Rach, "On the Adomian (decomposition) method and comparisons with Picard's method," Journal of Mathematical Analysis and Applications, vol. 128, no. 2, pp. 480-483, 1987.

[31] V. M. Soundalgekar, M. Singh, and H. S. Takhar, "MHD free convection past a semi-infinite vertical plate with suction and injection," Nonlinear Analysis: Theory, Methods \& Applications, vol. 7, no. 9, pp. 941-944, 1983.

[32] A. M. Lyapunov, General Problem on Stability of Motion, Taylor \& Francis, London, UK, 1992.

[33] S. Liao, Beyond Perturbation: Introduction to Homotopy Analysis Method, Chapman \& Hall/CRC, Boca Raton, Fla, USA, 2003.

[34] J.-H. He, "Homotopy perturbation technique," Computer Methods in Applied Mechanics and Engineering, vol. 178, no. 3-4, pp. 257-262, 1999.

[35] J.-H. He, "A coupling method of a homotopy technique and a perturbation technique for non-linear problems," International Journal of Non-Linear Mechanics, vol. 35, no. 1, pp. 37-43, 2000.

[36] S. J. Liao, "Comparison between the homotopy analysis method and homotopy perturbation method," Applied Mathematics and Computation, vol. 169, no. 2, pp. 1186-1194, 2005.

[37] M. Turkyilmazoglu, "Is homotopy perturbation method the traditional Taylor series expansion," Hacettepe Journal of Mathematics and Statistics, vol. 44, no. 3, pp. 651-657, 2015.

[38] T. Hayat and M. Sajid, "Analytic solution for axisymmetric flow and heat transfer of a second grade fluid past a stretching sheet," International Journal of Heat and Mass Transfer, vol. 50, no. 1-2, pp. 75-84, 2007.

[39] T. Hayat, Z. Abbas, M. Sajid, and S. Asghar, "The influence of thermal radiation on MHD flow of a second grade fluid," International Journal of Heat and Mass Transfer, vol. 50, no. 5-6, pp. 931-941, 2007.

[40] T. Hayat and M. Sajid, "Homotopy analysis of MHD boundary layer flow of an upper-convected Maxwell fluid," International Journal of Engineering Science, vol. 45, no. 2-8, pp. 393-401, 2007.

[41] S. Abbasbandy, "The application of homotopy analysis method to nonlinear equations arising in heat transfer," Physics Letters. A, vol. 360, no. 1, pp. 109-113, 2006.

[42] S. Abbasbandy, "Homotopy analysis method for heat radiation equations," International Communications in Heat and Mass Transfer, vol. 34, no. 3, pp. 380-387, 2007.

[43] M. Q. Brewster, Thermal Radiative Transfer and Properties, John Wiley \& Sons, 1992.

[44] H. S. Takhar, "Hydromagnetic free convection from a at plate," Indian Journal of Physics, vol. 45, pp. 289-311, 1971. 
[45] H. S. Takhar and G. Nath, "Similarity solution of unsteady boundary layer equations with a magnetic field," Meccanica, vol. 32, no. 2, pp. 157-163, 1997.

[46] H. Schlichting and K. Gersten, Boundary Layer Theory, Springer, Berlin, Germany, 2000.

[47] M. Turkyilmazoglu, "An effective approach for evaluation of the optimal convergence control parameter in the homotopy analysis method," Filomat, vol. 30, no. 6, pp. 1633-1650, 2016.

[48] S. Liao, Advances in the Homotopy Analysis Method, World Scientific, Singapore, 2014. 


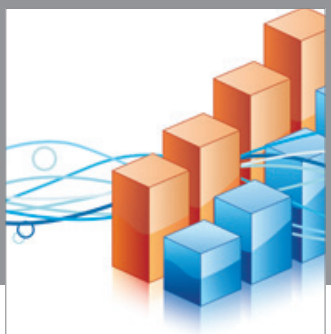

Advances in

Operations Research

vatem alat4

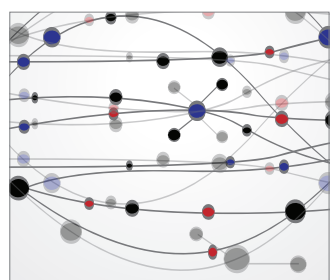

\section{The Scientific} World Journal
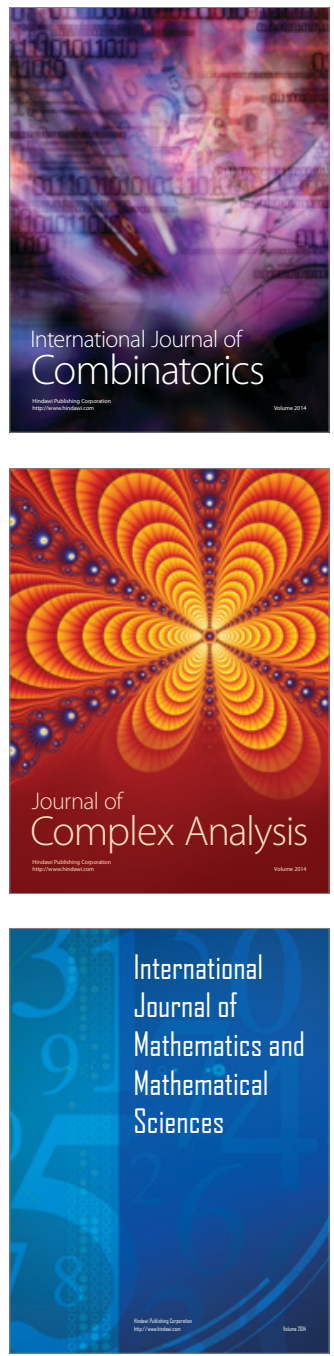
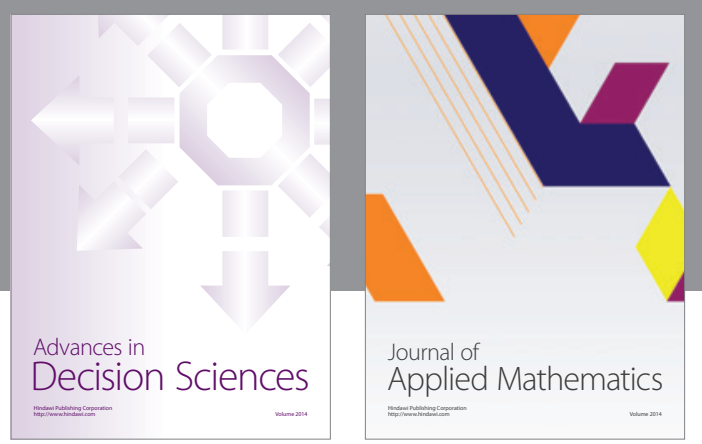

Algebra

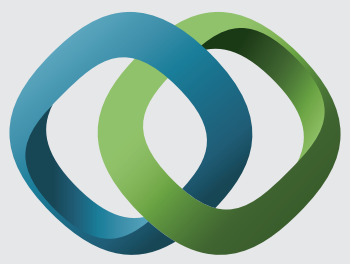

\section{Hindawi}

Submit your manuscripts at

http://www.hindawi.com
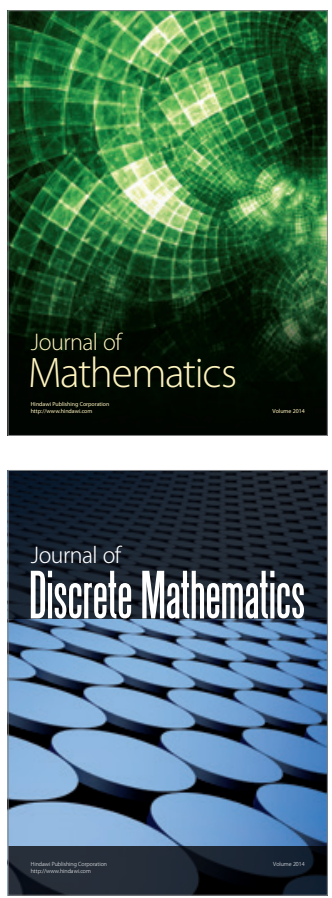

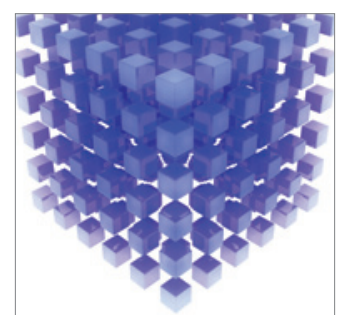

Mathematical Problems in Engineering
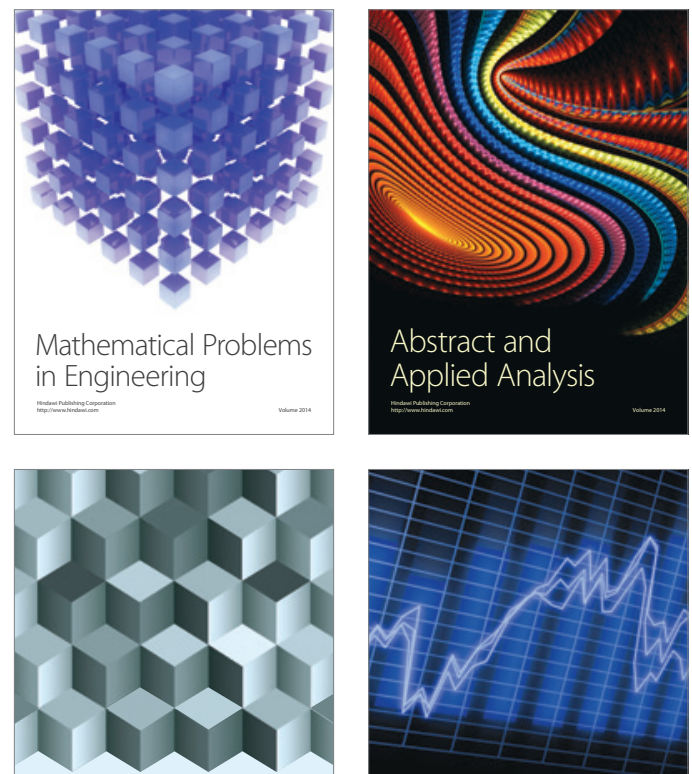

Journal of

Function Spaces

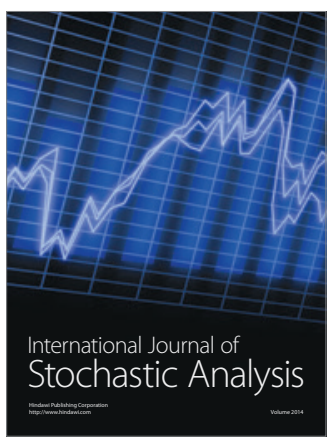

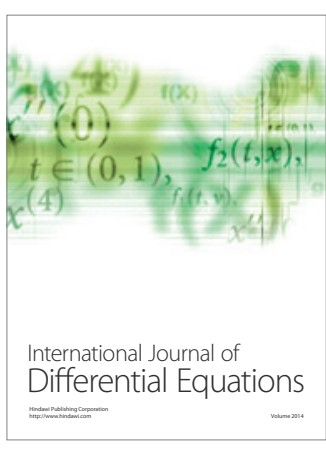
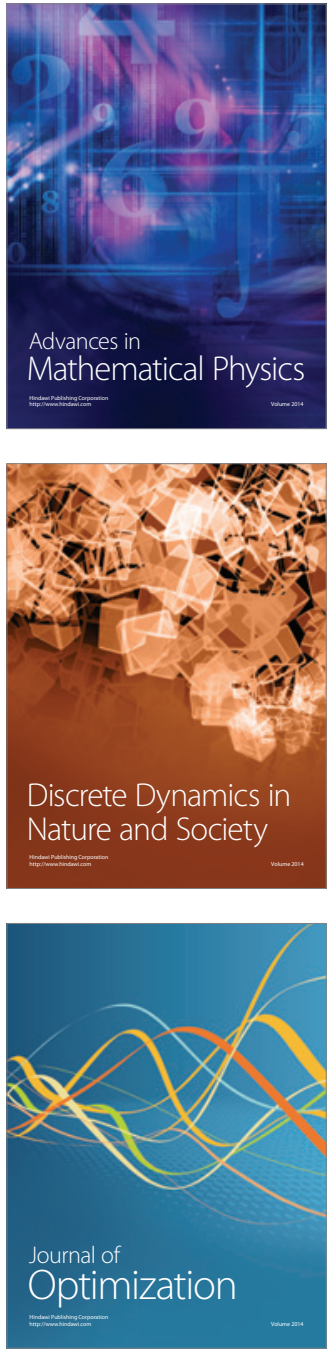